\title{
Truck and Trailer Routing Problem under fuzzy environment
}

\author{
Isis Torres ${ }^{1}$ Alejandro Rosete ${ }^{1}$ Carlos Cruz ${ }^{2}$ José L. Verdegay $^{2}$ \\ ${ }^{1}$ Department of Software Engineering, \\ ${ }^{1}$ Higher Polytechnic Institute José Antonio Echeverría, 11500 Havana, Cuba \\ ${ }^{2}$ Department of Computer Science and Artificial Intelligence \\ ${ }^{2}$ University of Granada, 18071 Granada, Spain
}

\begin{abstract}
The Truck and Trailer Routing Problem, TTRP, is an extension of the well-known Vehicle Routing Problem. This problem consist of a heterogeneous fleet composed of trucks and trailers to serve a set of customers. Most of models used in the literature assume that the data available are accurate, but in many practical problems the available knowledge about some data and parameters of the model involving uncertainty. In this study, we propose the application of a Soft Computing-based method to model and solve TTRP when the set of constrains is imprecise.
\end{abstract}

Keywords: TTRP, Fuzzy Linear Programming, Soft Computing, fuzzy constraints, fuzzy coefficients

\section{Introduction}

According to data from the Federal Highway Administration of United States of America approximately 11 million trucks moved 16.1 billion tons of freight worth 14.9 trillion only in 2011. In Australia, interstate road freight is expected to increase by 130 percent between 2008 and 2030 according to the Bureau of Infrastructure, Transport and Regional Economics. This information is a sample of the importance of the movement of freight by roads for many countries' current competitiveness in the global marketplace.

The strategies, that help to reduce costs and improve transport services, necessarily include the use of systems optimization. The Truck and Trailer Routing Problem (TTRP) is an important problem in this field, with a growing interest by its practical relevance in many real world problems such as milk collection [7], [18], [19], food distribution [16], [27] and postal delivery [1].

But in a real world these problems are very complex and the information is not always available with sufficient precision and completeness as desired for adequate planning and management. For example, in many circumstances it is usual that when we ask for the customer demand, the expressions are "about 50 units" or "no more than 65 units" etc. And the same can happen with the rest of the parameters that define the problem such as the travel times ("around 2-2:30 hours"), vehicle capacity ("no less than 3000 kgs", "about 100 kgs extra load"), etc.

The application of the fuzzy logic is a recognized way to describe mathematically all these situations. Unfortunately until now no much research has been conducted in this problem using this point of view. With this in mind, the goal of this paper is to model and solve the TTRP when the decision-maker is willing to permit some violations in the accomplishment of constraints and coefficients of constraints.

Thus, the paper is organized as follows. Section 2 presents a definition of TTRP and a review of the relevant literature on this problem. Section 3 proposes the model for TTRP with the set of constraints totally fuzzy and describes a general approach for solving it. Section 4 describes the implementation of the algorithm used. Results of the computational experiments are discussed in Section 5. Finally, conclusions are presented in the Section 6.

\section{Literature review and definition of the problem}

The TTRP consists in designing the optimal set of routes to serve a given set of customers, who are serviced by a fleet of vehicles with known capacity. A vehicle in the TTRP is composed by truck pulling a trailer and both are used for transporting goods. A truck plus a trailer is called a complete vehicle, and a vehicle without a trailer is called a pure truck. However, due to practical constraints, including government regulations, limited maneuvering space at customer site, road conditions, etc., some customers may only be serviced by the truck. Therefore, TTRP considers two different kinds of customers: a customer who is accessible with or without a trailer is called a vehicle customer (VC) and one who is only accessible without a trailer is called a truck customer (TC).

A route is defined as a vehicle route if the assigned vehicle is a complete vehicle, otherwise the route is referred to as a truck route since it is serviced by a truck. Routes originating from and terminating at a central depot, and they are limited by the capacity of vehicle used. Then, three types of routes can be 
defined in a TTRP solution:

- Complete Vehicle Route (CVR): Consisting of a main tour traveled by a complete vehicle, and at least one sub-tour traveled by the truck alone.

- Pure Truck Route (PTR): This type of route is traveled by a truck alone and are visited both customer type.

- Pure Vehicle Route (PVR): It is the tour traveled by a complete vehicle and contains only vehicle customer without any sub-tour.

Problems with characteristics similar to the TTRP but using other denominations were modeled in some previous works. Thus, Semet and Taillard [27] in 1993 presented an approach to solve a problem of one of the major chain stores in Switzerland. Later, Semet in 1995 extended the VRP taking into account the partial accessibility constraint introduced in their previous work [28].

The term "Truck and Trailer Routing Problem" was first used in 2002 by Chao [9] using a 2-phase approach. In the first phase a construction method was used to obtain an initial solution. In the second phase a Tabu Search (TS) algorithm was used to improve this solution. Scheuerer in 2006 [26] applied a TS approach to address the same problem. Two new construction heuristics as multi-start procedures are developed in this case. The first constructive heuristic, T-Cluster, prioritizes the construction of routes of complete vehicle and the TSweep heuristic, feasible routes are constructed by rotating a ray centered at the depot and customers are gradually added to the current route. In 2009, Lin et al. in [21] developed a very effective Simulated Annealing (SA) based heuristic to the TTRP with three neighborhoods. This proposal uses an indirect representation of the solution using a permutation the customers with additional artificial depot (dummy) to separate routes and terminate subtours. Caramia in 2010 [6] designed a mathematical programming based heuristic that also employs the cluster-first route-second approach. Villegas et al. in 2011 [34] solved the TTRP with a hybrid metaheuristic based on a Greedy Randomized Adaptive Search Procedures (GRASP), Variable Neighborhood Search (VNS) and Path Relinking (PR). This paper uses a route-first and cluster-second procedure for the randomized construction of GRASP.

Recently, a heuristic approach [12] was applied to the TTRP which combined local search and large neighborhood search as well as standard metaheuristics control strategies. Also, [35] proposed a two phase metaheuristic that uses the routes of the local optima of a hybrid GRASP $\times$ ILS (Iterated Local Search) as columns in a set-partitioning formulation of the TTRP. Lastly, a revision of different methods used for this problem is presented in [31].

Using the Chao definition [9], the TTRP can be formally defined on an undirected graph $G=(V, A)$, where $V=\{0,1,2, \ldots, n\}$, is a vertex set and $A=$ $\left\{\left(v_{i}, v_{j}\right): v_{i}, v_{j} \in V, i \neq j\right\}$ is the set of edges. The vertices represent customer, except the vertex $v_{0}$ that be corresponds to central depot. Each vertex of $V_{0}$ has a non-negative demand $d_{i}$. The access constraints create a partition of $V$ into two subsets: the subset of truck customer $\left(V_{T}\right)$ accessible only by truck and the subset of vehicle customer $\left(V_{V}\right)$ accessible either by truck or by truck with trailer.

$C$ is a matrix of non-negative cost. Each edge $(i, j)$ is associated with a cost $c_{i j}$ that represents the travel time required on the edge or the travel distance between vertex $i$ and vertex $j$. A heterogeneous fleet composed of $m_{c}$ trucks and $m_{r}$ trailers $\left(m_{c} \geq m_{r}\right)$ serves a set of customers. The capacities of the trucks and the trailers are $q_{c}$ and $q_{r}$ respectively. A route in the TTRP is composed of a partition of $V: R_{1}, R_{2}, \ldots, R_{m}$ and a permutation of $\delta_{m}$ specifying the order of the customers on route. Each route originating from and terminating at a central depot: $R_{m}=v_{0}, v_{1}, v_{2}, \ldots, v_{n+1}$, where $v_{0}=v_{n+1}$ denotes the depot. Each route is classified as route of complete vehicle $\left(R_{C V}\right)$ or route of pure truck $\left(R_{P T}\right)$. The goal of the TTRP is to find a set of least cost vehicle routes, one for each vehicle, such that each customer is visited exactly once, every route originates and ends at depot, and the capacity constraints are satisfied.

\section{Fuzzy approach for the Truck and Trailer Routing Problem}

TTRP is an optimization problem which by its nature favors the presence of uncertainty, and it is not always possible to have all the necessary information. In these circumstances Soft Computing methodologies has shown great potential in formulating and solving mathematical programming and decision making problems in the real world which are complex, ill-defined and not well understood.

But, the application of these techniques on this problem have not been much approached unfortunately until now. In this paper, we deal with a TTRP where the decision maker defines the maximum violation in the accomplishment of the set of constraints.

The problem can be represented as:

$$
\begin{array}{ll}
\max & \mathbf{y}=\mathbf{c x} \\
\text { s.t. } & \tilde{\mathbf{a}}_{\mathbf{i}} \mathbf{x} \leq^{f} \tilde{b}_{i} \\
& \mathbf{x} \geq \mathbf{0}, \quad i \in M=\{1, \ldots, m\}
\end{array}
$$

Verdegay [32], presented a general parametric approach to solve the TTRP with fuzzy constraints, which is composed of two phases: the first one transforms the fuzzy problem into several parametric problems in which a parameter $\alpha$-cut represents the decision maker's satisfaction level. Then, in the second phase each of these $\alpha$-problems is solved by 
means of classical optimization techniques. The obtained results, to the different $\alpha$ values, generate a set of solutions and then all of these particular $\alpha$ solutions are integrated by the Representation Theorem for fuzzy sets.

Later Delgado et al.[11] based on the last work develop a general solution strategy for fuzzy linear programming problems which involve fuzziness both in the coefficients and in the accomplishment of the constraints. Some examples of the application of these appriaches to VRP are: [2], [3], [4], [24]. Also, extensions to the quadratic programming models appear in [10], [29].

Delgado et al.[11] in this work used a ranking function that defines a mapping between a fuzzy number set and a classical numbers set. Thus, the set of constraints from Problem (1) can be modeled as follows:

$$
\mathcal{R}\left(\tilde{\mathbf{a}}_{\mathbf{i}}\right) \mathbf{x} \leq \mathcal{R}\left(\tilde{b}_{i}\right)+\mathcal{R}\left(\tilde{\tau}_{i}\right)(1-\alpha)
$$

where $\tau_{i}, i \in M=\{1, \ldots, m\}$, is a fuzzy number that represents the allowable tolerance for each constraint function, which is selected by the decision maker according his/her interests, $\alpha \in[0,1]$ is the satisfaction level, and the function $\mathcal{R}$ represents the ranking function. On this way a fuzzy solution to the problem can be obtained as:

$$
\begin{array}{cl}
\max & \mathbf{y}=\mathbf{c x} \\
\text { s.t. } & \mathcal{R}\left(\tilde{\mathbf{a}}_{\mathbf{i}}\right) \mathbf{x} \leq \mathcal{R}\left(\tilde{b}_{i}\right)+\mathcal{R}\left(\tilde{\tau}_{i}\right)(1-\alpha) \\
& \mathbf{x} \geq \mathbf{0}, \quad i \in M=\{1, \ldots, m\}
\end{array}
$$

Following the work of Campos and Verdegay [5] we use different conventional parametric models from which proper fuzzy solutions to 2 will be obtained for the following ranking functions used in the paper: three indexes proposed by Yager $[36,37,38]$, and the index used by Chang [8].

\section{Description of the algorithm implemented}

Since TTRP is a combinatorial optimization problem and it is computationally more difficult to solve compared with the VRP. Therefore, we decided to use an algorithm based on local search (in this case a Hill Climbing Method [30]), which is available from the BiCIAM library [15]. This algorithm belongs to the family of single-solution based metaheuristics and it starts at a given initial solution. At each iteration, the heuristic replaces the current solution by a neighbor that improves the objective function. The search stops when is performed a number of iterations.

Our implementation is based on the heuristic model proposed by [21]. This model can be described as:
- Solution representation: A solution is represented by a string of numbers consisting of a permutation of n customers and $N_{\text {dummy }}$ zeros (artificial depot), followed by the service vehicle types of individual VCs. The $N_{d u m m y}$ zeros are used to separate routes or terminate a sub-tour. The service vehicle type of a VC determines the type of the vehicle used to service the $\mathrm{VC}$. If the $\mathrm{VC}$ is serviced by a complete vehicle, its service vehicle type is set to be 0 . Otherwise, it is serviced by a truck alone is set to be 1 .

- Initial solution: The initial solution is randomly generated. It is comprised of a randomly ordered sequence of the customers and the dummy zeros, and randomly set service vehicle types of VCs.

- Neighborhood: A random neighborhood structure that includes insertion, swap, and change of service vehicle type. Also, we include an operator that inverts a subset of elements of the solution. We define the set $N(X)$ to be the set of solutions neighboring a solution $X$. In each iteration, the next solution $Y$ is generated from $N(X)$ either by any of the operators mentioned above as follows.

- Insertion: Position-based operator where an element at one position is removed and put at another position. Both positions are randomly selected [30].

- Swap: Order-based operator where arbitrarily is selected two elements and are swapped [30].

- Invert: Order-based operator where two elements are randomly selected and the sequence of elements between these two elements are inverted [30].

- Change of service vehicle type: This operator is performed by randomly selecting a $\mathrm{VC}$ and changing its service vehicle type from 1 to 0 or vice versa.

- Evaluation function: A process of decoding is used for evaluate the solution. In this process has been determined the customers on each route and the service vehicle type of each VC. Once this is done, the objective value (total distance traveled) is calculated as the sum of the distances of all routes. A penalty strategy was applied, which involves adding a penalty cost to the objective function for each extra vehicle requiring the solution. In these experiments the penalty cost associated with the number of extra trucks and trailers used is equal to 125 .

\section{Computational experiments}

In order to illustrate the efficiency and robustness of the approach used to solve the problem we present and discuss in this section the computational experiments carried out. 
We ran the experiments on a computer with an Intel Xeon running at $2.40 \mathrm{GHz}$ under Linux Ubuntu (version 3.2 .0 on 64 bit x $86 \mathrm{SMP}$ ) with $23 \mathrm{~GB}$ of RAM. We performed 30 independent runs with 100000 fitness evaluations used and the implementation of the algorithm Hill Climbing [30] of the library BiCIAM [15].

Instances of TTRP were solved for $\alpha \in\{0.0,0.1$, $0.2,0.3,0.4,0.5,0.6,0.7,0.8,0.9,1.0\}$. The amount demand of each customer and the limited vehicle capacities are triangular fuzzy numbers. The triangular fuzzy numbers were obtained in the form of $10 \%$ variation in the modal value in each case, e.g. the truck capacity is equal 100 vary up to 10 units, then $q_{c}=(90,100,110)$. Also, we used four ranking function (see Table 1):

\begin{tabular}{cl}
\hline \multicolumn{1}{c}{ Index } & Code \\
\hline First Index Yager & FIY \\
Second Index Yager & SIY \\
Third Index Yager & TIY \\
Chang Index & CI \\
\hline
\end{tabular}

Table 1: Indexes used.

\begin{tabular}{ccccl}
\hline Customers & VC & TC & Trucks & Trailers \\
\hline 50 & 25 & 25 & $5(100)$ & $3(100)$ \\
\hline
\end{tabular}

Table 2: TTRP benchmark data.

In order to test our model, we use the second instance of testbed described by Chao 2002 [9]. Table 2 shows the characteristics of selected problem, in which $\mathrm{VC}$ is a vehicle customer, $\mathrm{TC}$ is a truck customer, Trucks is the number of trucks and their capacity, and Trailers is the number of trucks and their capacity.

Table 3 reports the best (Best Cost) and average (Avg.) cost over 30 runs for each ranking function in test problems. Note that there are some similar best cost values to different $\alpha$-values in each Index. This situation is possible because the violation value of the capacity could not be sufficient to improve the cost of objective function and therefore the best cost value could not change and keep the previous value of $\alpha$-cut.

We can see that the proposal shows its efficiency for the different comparison indexes used in this work. Thus, any index that satisfies the properties from [38] can be used with this method.

\section{Conclusions and future research}

Truck and Trailer Routing Problem is an emergent and interesting problem which inevitably involves a degree of uncertainty or imprecision due to the lack of knowledge when it is modeled in the realworld. In contrast to what happens in the fuzzy formulation for Vehicle Routing Problems, unfortunately to date, not much research has been done in this important class of problem. For this reason it would be appropriate to focus research toward defining TTRP models for incorporating the uncertainty present in the data, and hence it makes perfect sense to think about Soft Computing techniques.

In this paper a known fuzzy parametric approach is proposed as a general method to solve this problem with fuzzy constraints and fuzzy coefficients. Thus, each $\alpha$-solution is integrated in a fuzzy solution and the decision maker would have a set of solutions according to their satisfaction degrees. The proposal was validated using a known benchmark for this problem.

The authors aim to extend this research in future works in order to try to solve different point of view of this kind of problem. Also, all these models and methods will be used to solve real and practical business problems.

\section{Acknowledgements}

This work was supported in part by the projects TIN2011-27696-C02-01 from the Spanish Ministry of Economy and Competitiveness, P07-TIC-02970 from the Andalusian Government, and GENILPYR-2014-9 from CEI BioTIC Granada, University of Granada.

\section{References}

[1] Bodin, L. \& Levy, L. Scheduling of local delivery carrier routes for the United States postal service. In Arc Routing: Theory, Solutions, and Applications, 419-442 (2000)

[2] Brito, J., Martï $\frac{1}{2}$ nez, F. J., Moreno., J. A. \& Verdegay, J. L. A GRASP-VNS Hybrid for the Fuzzy Vehicle Routing Problem with Time Windows. In Proceedings of the Computer Aided Systems Theory - EUROCAST 2009, Lecture Notes in Computer Science 5717, 825-832 (2009)

[3] Brito, J., Martï $\frac{1}{2}$ nez, F. J., Moreno., J. A. \& Verdegay, J. L. ACO-GRASP-VNS Metaheuristic for VRP with Fuzzy Windows Time Constraints. In Proceedings of the $13^{\text {th }}$ International Conference on Computer Aided Systems Theory - EUROCAST 2011, Part I Lecture Notes in Computer Science 6927, 440-447 (2012) Proceedings

[4] Brito, J., Moreno., J. A. \& Verdegay, J. L. Transport route planning models based on fuzzy approach. Iranian Journal of Fuzzy Systems, 9(1), 141-158 (2012)

[5] Campos, L. \& Verdegay, J.L. Linear programming problems and ranking of fuzzy numbers. Fuzzy Sets and Systems, 32(7), 1-11 (1989)

[6] Caramia, M. \& Guerriero, F. A heuristic approach for the truck and trailer routing problems. Journal of the Operational Research Society, 61(7), 1168-1180 (2010) 


\begin{tabular}{ccccccccc}
\hline & $\begin{array}{c}\text { First Index Yager } \\
\text { (FIY) }\end{array}$ & \multicolumn{2}{c}{$\begin{array}{c}\text { Second Index Yager } \\
\text { (SIY) }\end{array}$} & \multicolumn{2}{c}{$\begin{array}{c}\text { Third Index Yager } \\
\text { (TIY) }\end{array}$} & \multicolumn{2}{c}{$\begin{array}{c}\text { Chang Index } \\
\text { (CI) }\end{array}$} \\
$\alpha$ & Best Cost & Avg. & Best Cost & Avg. & Best Cost & Avg. & Best Cost & Avg. \\
\hline $\mathbf{0 . 0}$ & 736.92 & 812.29 & 527.49 & 594.21 & 731.65 & 799.63 & 696.21 & 788.80 \\
$\mathbf{0 . 1}$ & 727.72 & 810.76 & 530.65 & 594.89 & 731.65 & 802.35 & 703.60 & 800.46 \\
$\mathbf{0 . 2}$ & 727.72 & 811.28 & 530.65 & 594.89 & 731.65 & 809.26 & 712.32 & 810.72 \\
$\mathbf{0 . 3}$ & 731.24 & 815.30 & 530.65 & 594.89 & 731.65 & 812.40 & 732.38 & 817.27 \\
$\mathbf{0 . 4}$ & 744.20 & 823.58 & 530.65 & 596.75 & 731.65 & 817.09 & 732.38 & 825.68 \\
$\mathbf{0 . 5}$ & 744.20 & 831.69 & 530.65 & 596.81 & 758.19 & 837.16 & 760.45 & 850.07 \\
$\mathbf{0 . 6}$ & 744.20 & 837.88 & 533.43 & 600.16 & 753.62 & 837.53 & 762.05 & 874.21 \\
$\mathbf{0 . 7}$ & 750.49 & 867.13 & 539.74 & 600.48 & 758.89 & 850.22 & 771.80 & 888.54 \\
$\mathbf{0 . 8}$ & 791.40 & 880.70 & 530.65 & 601.27 & 758.89 & 853.22 & 787.01 & 909.32 \\
$\mathbf{0 . 9}$ & 791.40 & 889.28 & 539.74 & 603.30 & 776.85 & 867.77 & 830.25 & 936.71 \\
$\mathbf{1 . 0}$ & 822.07 & 905.22 & 539.74 & 604.63 & 786.78 & 894.88 & 830.25 & 949.60 \\
\hline
\end{tabular}

Table 3: Results obtained with each ranking function in TTRP instances.

[7] Caramia, M. \& Guerriero, F. A Milk Collection Problem with Incompatibility Constraints. Interfaces, 40(2), 130-143 (2010)

[8] Chang, W. Ranking of fuzzy utilities with triangular membership functions. Proc. Int. Conf. on Policy Anal. and Inf. Systems 263272 (1981)

[9] Chao, I-M. A tabu search method for the truck and trailer routing problem. Computers $\mathscr{G} O \mathrm{p}$ erations Research, 29(1), 33-51 (2002)

[10] Cruz, C., Silva, R. C. \& Verdegay, J. L. Extending and relating different approaches for solving fuzzy quadratic problems. Fuzzy Optimization and Decision Making, 10(3), 193-210 (2011)

[11] Delgado, M., Verdegay, J. L. \& Vila, M. A. A general model for fuzzy linear programming. Fuzzy Sets and Systems, 29(1), 21-29 (1989)

[12] Derigs, U., Pullmann, M. \& Vogel, U. Truck and trailer routing-problems, heuristics and computational experience. Computers \& Operation Research, 40(2), 536-546 (2013)

[13] Drexl, M. A branch and price algorithm for the truck and trailer routing problem. Technical Report, N. 27/2006 Deutsche Post Endowed Chair of Optimization of Distribution Networks, RWTH Aachen University, Germany (2007)

[14] Drexl, M. Branch and price and heuristic colum generation for the generalized truck and trailer routing problem. Revista de Metodos Cuantitativos para la Economia y la Empresa, 12, 5-38 (2011)

[15] Fajardo, J.\& Rosete, A. Algoritmo multigenerador de soluciones para la competencia y colaboracion de generadores metaheuristicos. $R e$ vista Internacional de Investigacion de Operaciones (RIIO). 1, (2011)

[16] Gerdessen, J. C. Vehicle routing problem with trailers. European Journal of Operational Research, 93(1), 135-147 (1996)

[17] Gillett, B. E. \& Miller, L. R. A heuristic algo- rithm for the vehicle-dispatch problem. Operations research, 22(2), 340-349 (1974)

[18] Hoff, A. \& Lokketangen, A. A tabu search approach for milk collection in Western Norway using trucks and trailers, In Proceedings of the Sixth Triennial Symposium on Transportation Analysis, Phuket, Thailand (2007)

[19] Hoff, A. Milk collection in western Norway using trucks and trailers, In Proceedings of the $5^{\text {th }}$ International Workshop on Freight Transportation and Logistics (ODYSSEUS 2012), Mykonos, Greece, 180-183(2012)

[20] Lin, S. Computer solutions to the traveling salesman problem. Bell System Technical Journal 44(10), 2245-2269 (1965)

[21] Lin, S-W., Yu, V. F. \& Chou, S-Y. Solving the truck and trailer routing problem based on a simulated annealing heuristic. Computers \&6 Operations Research, 36(5), 1638-1692 (2009)

[22] Lin, S-W., Yu, V. F. \& Chou, S-Y. A note on a the truck and trailer routing problem. Expert Systems with Applications, 37(1), 899-903 (2010)

[23] Lin, S-W., Yu, V. F. \& Lu,C-C. A simulated annealing heuristic for the truck and trailer routing problem with time windows. Expert Systems with Applications, 38(12), 15244 15252 (2011)

[24] Melian, B. \& Verdegay, J. L. Using fuzzy numbers in network design optimization problems. IEEE Transactions On Fuzzy Systems, 19(5), 797-806 (2011)

[25] Or, I. Traveling salesman-type combinatorial optimization problems and their relation to the logistics of regional blood banking, Dissertation Department of Industrial Engineering and Management Science, Northwestern University, Evanston (1976)

[26] Scheuerer, S. A tabu search heuristic for the truck and trailer routing problem. Computers \& Operations Research, 33(4), 894-909 (2006)

[27] Semet, F. \& Taillard, E. Solving real-life ve- 
hicle routing problems efficiently using tabu search. Annals of Operations Research, 41(4), 469-488 (1993)

[28] Semet, F. A two-phase algorithm for the partial accessibility constrained vehicle routing problem. Annals of Operations Research, 61(1), 4565 (1995)

[29] Silva, R. C., Cruz, C.\& Verdegay, J. L. Fuzzy costs in quadratic programming problems. Fuzzy Optimization and Decision Making, 12(3), 231-248 (2013)

[30] Talbi, E-G. Metaheuristics: from design to implementation, 500 pp. John Wiley and Sons, New Jersey (2009)

[31] Torres, I., Verdegay, J. L., Cruz, C. \& Rosete, A. Models and solutions for truck and trailer routing problems: An overview. International Journal Applied Metaheuristic Computing, 4(2), 31-43 (2013)

[32] Verdegay, J. L. Fuzzy mathematical programming. In Fuzzy Information and Decision Processes (Eds. Gupta, M.M., Sanchez, E.), 231237 (1982)

[33] Villegas, J. G., Prins, C., Prodhon, C., Medaglia, A. L. \& Velasco, N. GRASP/VND and multi-start evolutionary local search for the single truck and trailer routing problem with satellite depots. Engineering Applications of Artificial Intelligence, 23(5), 780-794 (2010)

[34] Villegas, J. G., Prins, C., Prodhon, C., Medaglia, A. L. \& Velasco, N. A GRASP with evolutionary path relinking for the truck and trailer routing problem. Computers $\& 5$ Operations Research, 38(9), 1319-1334 (2011)

[35] Villegas, J. G., Prins, C., Prodhon, C., Medaglia, A. L. \& Velasco, N. A matheuristic for the truck and trailer routing problem. European Journal of Operational Research, 230(2), 231-244 (2013)

[36] Yager, R.R. Ranking fuzzy subsets over the unit interval. Proc. 1978 CDC 1435-1437 (1978)

[37] Yager, R.R. On choosing between fuzzy subsets, Kybernetes 9, 151-154 (1980)

[38] Yager, R.R. A procedure for ordering fuzzy subsets of the unit interval. Inform Sci. 24, 143-161 (1981) 